\title{
Shifting the Paradigm of Routine Blood Draws
}

Owen Brown, MD; Jennifer Angell, MD*; Shaina Sekhri, MD;

Sagardip Majumder, MS; Rachel Wallace, BS; Chandu Vemuri, MD

Please note that there are 3 co-first authors:

Owen Brown \& Jennifer Angell \& Shaina Sekhri.

\section{ABSTRACT}

Every day, people are found debilitated or unconscious with unknown cause. Currently, the full diagnostic workup does not begin until the patient enters the hospital, delaying a critical treatment window. Point-of-care testing is an exploding field with a projected value of $\$ 36.96$ billion by 2021. Notable products include glucose monitoring, drugs-ofabuse testing, and cardiometabolic monitoring. While several point-of-care devices exist independently, use of multiple products is cumbersome and limits the ability to efficiently inform medical decision-making. No single technology provides a comprehensive analysis.

Po1nt is a portable, low-cost, handheld device equipped with an optical detection system that performs a wide-variety of blood tests. Single-use cartridges accompany the multi-use detection device. The disc-shaped cartridges have multiple channels to perform a variety of tests simultaneously utilizing fluorescent markers. The cartridges are situationally designed for use in a variety of common medical scenarios.
Author E-mail List:

owenb@umich.edu;

angellj@umich.edu;

ssekhri@umich.edu;

sagardip@umich.edu;

rpwall@umich.edu;

cvemuri@umich.edu

Conflicts of interest: Authors have no conflicts of interest.

*Corresponding Author: Jennifer Angell, (248) 882-4536, angellj@umich.edu

\section{Company Purpose}

he triage process and diagnostic workup often begin once a patient enters the hospital. This prolongs a critical window of time from discovery to appropriate treatment, which is vital in acute care. Currently, labs are only drawn after a patient reaches the emergency department, and they must often be sent to a central laboratory with the sample changing hands numerous times. The complex workflow of obtaining labs delays decision-guiding results up to an hour. Although several point-of-care devices exist independently, the use of multiple products is cumbersome and limits their utility in 


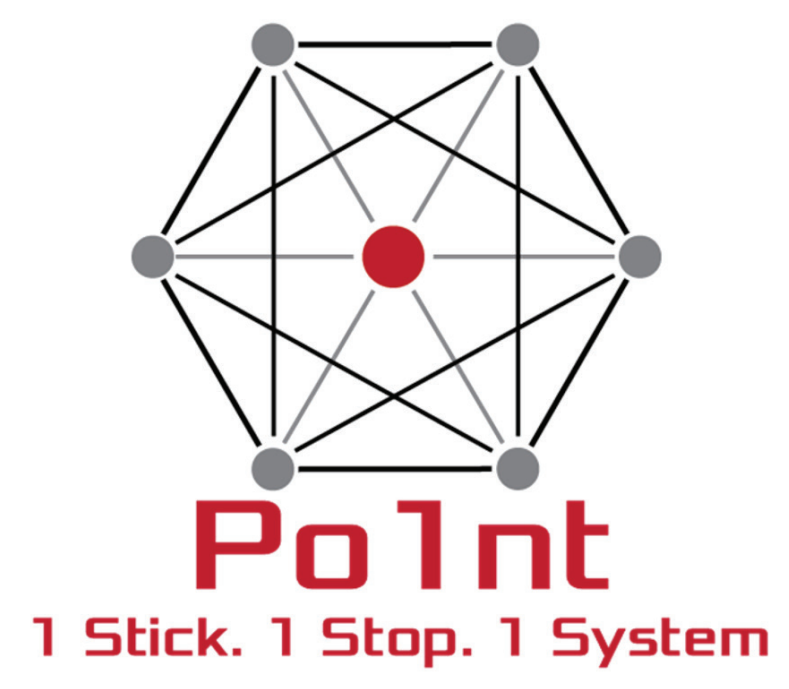

FIGURE 1. Po1nt logo

the acute setting. As of yet, no single technology provides a comprehensive analysis.

Po1nt is a unifying diagnostic system with the ability to run a large panel of tests crucial to decision-making within numerous medical settings. In an emergency medical service, lab values illuminating elevated cardiac enzymes, infection, or electrolyte imbalances would inform the care team prior to reaching the hospital, allowing for more efficient patient care and improved outcomes while saving critical time and money. A multipurpose point-of-care device would also decrease the volume of blood required for laboratory tests, which can further exacerbate a critically ill patient's health. ${ }^{1}$ Likewise, in a hospital's units, nurses could collect labs and results at the patient's bedside with Po1nt. The handheld device could determine a patient's hemoglobin or sodium level in simply seconds rather than blood draws being sent to the laboratory through complex processing.

Currently in the outpatient setting, patients often have labs drawn after seeing a provider. The results must subsequently be followed up by a patient's physician, who will modify care and medications accordingly. The extensive workflow introduces room for error, such as patients failing to visit the lab, results being missed by staff, or difficulty in contacting patients. Po1nt would streamline this workflow and eliminate the need to contract blood testing to outside laboratories, ultimately saving time for both the physician and patient, as well as providing another revenue stream for physicians. Po1nt's user-friendly interface with a one-touch start, interchangeable test cartridges, and direct communication with the electronic medical record would decrease costs, improve patient care, and streamline workflow across multiple medical care settings.

\section{Market Analysis}

There are a variety of market competitors including i-STAT Handheld Blood Analyzer, epoc ${ }^{\circledR}$ Blood Analysis System, General Fluidics, and Velano Vascular. There has not been significant uptake of any of these products due to a variety of engineering defects that impose obstacles to clinical application. Our team interviewed the director of respiratory care at Michigan Medicine who is responsible for the evaluation and introduction of point-of-care devices. The director noted that preexisting devices specifically require either cumbersome calibration, preprocessing of blood, extensive training, or very expensive detection methods. The primary downfall of Theranos involved their inability to produce laboratory results as advertised, instead relying on a central processing laboratory to perform the testing that was marketed as possible through their pointof-care system. ${ }^{2}$ Throughout our design process, we engineered Po1nt to specifically avoid these obstacles (Table 1).

Our product will be superior in its unique fluorescent-based detection method. Po1nt only requires a microliter of blood rather than 1-1.5 $\mathrm{mL}$, thus decreasing harm to patients from cumulative blood draws. We anticipate Po1nt's one-touch start function will require minimal training and operator calibration. An additional competitive advantage would be the competitive pricing of a Po1nt device and its cartridges, making lab tests more affordable for medical providers. 


\begin{tabular}{|c|c|c|c|c|c|c|}
\hline & Polnt & MicroEye@ & $\begin{array}{c}\text { i-STAT TM } \\
\text { Handheld } \\
\text { Blood Analyzer }\end{array}$ & $\begin{array}{c}\text { epoc@ Blood } \\
\text { Analysis } \\
\text { System }\end{array}$ & $\begin{array}{l}\text { General } \\
\text { Fluidics }\end{array}$ & $\begin{array}{l}\text { Velano } \\
\text { Vascular }\end{array}$ \\
\hline $\begin{array}{c}\text { Utilizes } \\
\text { preexisting } \\
\text { vascular access }\end{array}$ & & $X$ & X & $X$ & & \\
\hline Easy calibration & & $X$ & $X$ & $X$ & $X$ & $\mathbf{N} / \mathbf{A}$ \\
\hline $\begin{array}{c}\text { No preprocessing } \\
\text { of blood }\end{array}$ & & & X & $X$ & & $\mathbf{N} / \mathbf{A}$ \\
\hline $\begin{array}{c}\text { Inexpensive } \\
\text { detection method }\end{array}$ & & $X$ & & & $\boldsymbol{\lambda}$ & $\mathbf{N} / \mathbf{A}$ \\
\hline $\begin{array}{l}\text { Draws and tests } \\
\text { blood }\end{array}$ & & $X$ & $X$ & $X$ & & X \\
\hline
\end{tabular}

TABLE 1. Market Comparison

\section{Product}

Po1 nt is a handheld point-of-care device that performs context-specific blood testing and yields results in a matter of minutes. To best describe how Po1nt works, envision the Keurig and the K-cup model. The Keurig in this analogy would be our multi-use detection system, and the K-cup would be our single-use cartridge (Figure 2).
Po1nt can be connected directly to a preexisting IV using a specially designed adapter or an outpatient blood draw syringe. A battery will power an internal DC motor and its fluorescence-based detection system. Per our understanding, this fluorescence-based detection method is unique to the point-of-care market. Single-use, disc-shaped cartridges will be impregnated with appropriate

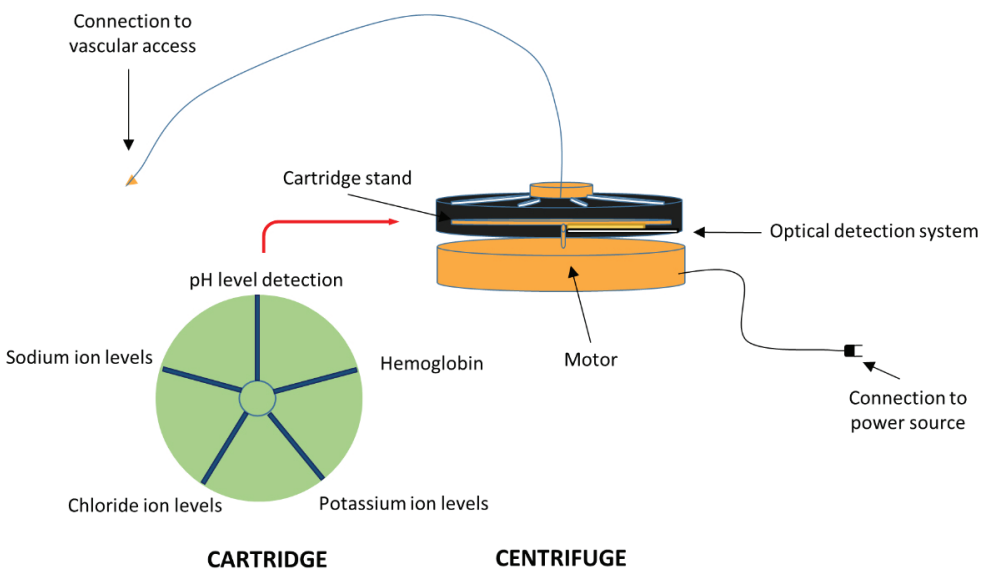

FIGURE 2. Po1nt Detection Schematic with Micro-fluidic Cartridge Design 
biomarkers that will detect concentrations of different metabolites currently analyzed in common laboratory tests. Each cartridge will be designed to have multiple channels pertaining to the array of tests desired in a specific setting. Each channel within the cartridge will be lined with metabolite specific biomarkers conjugated with certain fluorophores. This single-use cartridge will then be inserted into the detection device, analogous to inserting a K-cup into a Keurig machine.

Cartridges will be manufactured as a sealed vacuum so that when "Start" is pressed, a small needle punctures the cartridge, and the negative pressure within the cartridge will draw blood into the system. This process, combined with battery-powered centrifugation, will allow for the separation of blood into its different phases (cells and plasma) based on density differential and subsequent binding of the metabolites to their corresponding biomarkers. The result will be fluorescence activation of the conjugated fluorophores. An external LED light source and appropriate fluorescence sensors placed in the device housing will detect and quantify concentration measurements for all channels. Because optical mode of detection is proposed for this device, the cartridges will be made from transparent polymers. Custom cartridges can be designed as a suite of tests to optimize utility in a variety of medical contexts.

We plan to first replicate the complete blood count (CBC) and basic metabolic panel (BMP) due to those being the most prevalent tests in the clinical setting. Our first cartridge will measure calcium, sodium, potassium, and hydrogen ion levels as well as a red blood cell count. We plan to expand to detection of other important molecules that can inform the functioning of numerous vital organs. For example, we anticipate a renal panel for dialysis patients or a cardiac panel for acute coronary syndrome workup.

The detection sensor has been successfully developed and preliminary proof-of-concept testing has demonstrated calcium ion level detection within $7 \%$ accuracy. The detection device has been digitally rendered in computer-aided design (CAD); the development of a physical prototype is the next step after proof-of-concept testing.

\section{Business Model}

Po1nt's business model focuses on decreasing the cost of blood draws that currently require complex infrastructure and staffing to complete. For example, a BMP at Michigan Medicine costs $\$ 96.00$. Po1 nt could complete a BMP for $\$ 5$ at the patient's bedside. Anticipated production costs are $\$ 65$ per device and \$2.50 per cartridge, providing large profit margins. While saving hospitals money, Po1nt's inexpensive detection system would create revenue from the detection device as well as the single-use cartridges that each contain a certain panel of tests. The single-use cartridges would be a continual source of revenue for the company. We envision Po1nt being housed in ambulances, emergency departments, intensive care units, and outpatient clinics. The applications are endless: One stick, one stop, one system.

\section{Team Members and Background}

Chandu Vemuri, MD: Vemuri is a vascular surgeon at the University of Michigan whose research focuses on nanotherapeutics for patients with vascular disease. He is actively involved in the Michigan Medicine's Fast Forward Medical Innovation program and has worked with multiple student groups to translate their ideas to improve patient care into solutions.

Owen Brown, BS: Brown is a graduate of the University of Michigan Medical School who will be specializing in plastic surgery at Emory University. He is a member of Medical Innovation Path of Excellence and co-developed the first-ever Michigan Medical School Shark Tank competition. Outside of innovation, his research focuses on surgical outcomes and opioid prescribing.

Jennifer Angell, BS: Angell is a graduate of the University of Michigan Medical School who will be specializing in anesthesiology at Oregon Health 
and Science University. She has experience innovating in curriculum as well mobile applications, such as the design and development of the Stay in the Blue application sponsored by the University of Michigan University Health Services. Additionally, she designed a program to facilitate the delivery of healthcare to asylum seekers, which was highlighted by the Association of American Medical Colleges (AAMC) as one of the Transformative Student Initiated Innovations of 2016. Her research focuses on surgical outcomes and rootcause analyses in global settings.

Shaina Sekhri, MD: Sekhri is a graduate of the University of Michigan Medical School who will specialize in internal medicine at the Medical College of Wisconsin. She has led the University of Michigan's student chapter of the American Medical Women's Association (AMWA) as president, focusing on community engagement, domestic violence awareness, gender equity in the workplace, and mentorship. Her main research interest is the opioid epidemic.

Sagardip Majumder, BME, MMA: Majumder is pursuing a PhD in chemical engineering from the
University of Michigan. His research focus is fluid mechanics, particularly nano-fluidics, and developing biosensors.

Rachel Wallace, BSE: Wallace is pursuing a PhD in molecular engineering at the University of Chicago after completing her bachelor's in chemical engineering at the University of Michigan. Her research focuses on nanoparticle drug-delivery systems and technology to induce immunological tolerance for treating transplant rejection and autoimmune disorders.

\section{References}

1. McLenon M, Botz G, Phipps R, Clanton N. A quality improvement initiative to decrease the frequency of blood draws among critically ill patients. American Thoracic Society 2014 International Conference. https://www.atsjournals.org/doi/pdf/10.1164/ ajrccm-conference.2014.189.1_MeetingAbstracts .A4491. Accessed April 24, 2019.

2. Fiala C, Diamandis EP. The meteoric rise and dramatic fall of Theranos: lessons learned for the diagnostic industry. Clinical Chemistry and Laboratory Medicine (CCLM). 2018;56(9):1443-1446. doi:10.1515/cclm-2018-0353. 